\title{
Confocal microscopy study of the distribution, content and activity of mitochondria during Paracentrotus lividus development
}

\author{
GIOVANNI MORICI*, MARIA AGNELLO*, FILIPPO SPAGNOLO $\dagger$, \\ MARIA CARMELA ROCCHERI*, CARLO MARIA DI LIEGRO* \& \\ ANNA MARIA RINALDI* \\ *Dipartimento di Biologia Cellulare e dello Sviluppo 'A. Monroy', Università degli Studi di Palermo, \\ viale delle Scienze, ed. 16, 90128 Palermo, Italy \\ $†$ Dipartimento di Matematica e Applicazioni, Università degli Studi di Palermo, Palermo, Italy
}

Key words. Activity, development, CLSM, mitochondrial mass, sea urchin.

\begin{abstract}
Summary
In the present paper we applied confocal microscopy and fluorescence technologies for studying the distribution and the oxidative activity of sea urchin (Paracentrotus lividus) mitochondria during development, by in vivo incubating eggs and embryos with cell-permeant MitoTracker probes. We calculated, by a mathematical model, the intensity values, the variations of intensity, and the variation index of incorporated fluorochromes. Data demonstrate that mitochondrial mass does not change during development, whereas mitochondrial respiration increases. In addition, starting from 16 blastomeres stage, some regions of the embryo contain organelles more active in oxygen consumption.
\end{abstract}

\section{Introduction}

The confocal laser scanning microscope (CLSM) technology, commercially available since 1983, allows the observation of the inner site of living or fixed cells and tissues, and it is able to capture, through computer, serial optical sections with a good contrast, creating three-dimensional models. The development of confocal microscopy was accelerated by computer, laser and detector technologies, by interference filters and fluorochromes, as well as by highly specialized objectives.

CLSM allows researchers to collect a series of optical sections and, compared to the wide-field conventional microscopy, offers advantages as a much better control of depth of field and reduction or removal of background noise away from the focal plane. The CLSM technology, based on fluorescence emissions, uses probes able to bind to biological macromolecules,

Correspondence to: Anna Maria Rinaldi. Tel: +39091 6577408; fax: +39091 6577430; e-mail: rinaldi@unipa.it such as proteins or nucleic acids, localizing them into cell structures (like cytoskeleton, mitochondria, Golgi apparatus, endoplasmic reticulum or nucleus). Fluorescent probes are also used to focus environmental variables (like concentration of metallic ions, $\mathrm{pH}$, reactive oxygen species and membrane potential). This method allows studying cell integrity, apoptosis, endocytosis/exocytosis, traffic of proteins, signal transduction or enzymatic activities, and it is helpful in gene mapping and chromosome analysis. The commonly used lasers are sources of high-intensity monochromatic light, very useful for various microscopy techniques such as optical trapping, photobleaching and total internal reflection fluorescence. The CLSM has wide application in biological sciences, medical fields (Pawley, 1995; Zhang et al., 2000) and in marine biological studies, specifically concerning reproduction and development of marine invertebrates (Hertzler \& Clark, 1992; Summers et al., 1993; Holy, 1999, Buttino et al., 2003).

During sea urchin oogenesis, mitochondria are synthesized and accumulated in very high number. Mature eggs contain hundreds of thousands organelles, which are partitioned to the daughter cells after fertilization. It has been largely demonstrated that the number of maternal mitochondria is sufficient to support development until the larva stage. Hence, the total number of organelles does not increase during development (Matsumoto et al., 1974; Bresch, 1978; Rinaldi et al., 1979a,b). After fertilization, the respiratory rate of sea urchin embryos increases, then decreases, keeping quite constant up to blastula, slightly increases after hatching, it reaches the maximum value at gastrula stage and then it is maintained at a high level up to pluteus (see Giudice, 1985; Fujiwara \& Yasumasu, 1997).

It has been demonstrated that, in Strongylocentrotus purpuratus, mitochondria are asymmetrically distributed in unfertilized eggs and that the overall polarity, maintained in the 
zygote and during development, is responsible for the formation of the oral-aboral axis, in that the side of the embryo containing the highest density of mitochondria gives rise to the oral pole (Cameron et al., 1989; Davidson et al., 1998; Coffman et al., 2001, 2004).

In the present paper, we applied CLSM and fluorescence technologies to study the distribution and the oxidative activity of Paracentrotus lividus mitochondria during development by in vivo incubating eggs and embryos with cell-permeant MitoTracker probes. The probes are very useful tools for determining the mass [MitoTracker ${ }^{\mathrm{TM}}$ Green (MTG) FM] and the oxidative activity [MitoTracker ${ }^{\mathrm{TM}}$ Orange (MTO) CM$\mathrm{H}_{2}$ TMRos] of mitochondria. In order to interpret results and to draw unequivocal conclusions, we elaborated a mathematical discrete model calculating the intensity values of fluorochromes, the variations of intensity incremental ratio, and the variation index in the different developmental stages, overcoming the problems due to the geometry of the system.

\section{Materials and methods}

\section{Embryo culture}

Adult sea urchins of the Mediterranean species P. lividus were collected along Sicily's western coasts. Eggs were fertilized and embryos were grown at $18^{\circ} \mathrm{C}$, at the concentration of $5000 \mathrm{~mL}^{-1}$ in Millipore filtered sea water containing antibiotics (mfswa), in a beaker with a rotating propeller, until reaching the desired stages. Unfertilized eggs were collected in mfswa.

\section{Treatments}

To a suspension of eggs or embryos were added $200 \mathrm{nM}$ (f.c.) of MTG (FM M-7514) and $100 \mathrm{nM}$ (f.c.) of MTO CM- $\mathrm{H}_{2}$ (TMROS M-7511, Molecular Probes). After 60-min incubation at $18^{\circ} \mathrm{C}$ in a dark room, in rotation, the samples were washed several times with mfswa and fixed with $0.1 \%$ formaldehyde in sea water-Tris-HCl pH 8.0.

MTG, nonfluorescent in aqueous solutions, becomes fluorescent only when it accumulates in the mitochondrial lipid environment, regardless of membrane potential, thus representing a very useful tool for determining the mitochondrial mass. Furthermore this dye, more photostable than rhodamine 123, shows a blueshifted emission maximum (approximately $10 \mathrm{~nm}$ compared to the emission maximum of rhodamine 123). In conclusion, MTG produces a fluorescent staining pattern that should be better resolved than that of the red-fluorescent probes.

MTO CM- $\mathrm{H}_{2}$ TMRos, the nonfluorescent reduced form of tetramethylrosamine, once it enters an actively respiring cell, it is oxidized by the molecular oxygen. It is then sequestered by mitochondria, because it interacts with the thiol groups of peptides, forming an aldehydic mitochondrion-selective probe, fluorescent in the red spectrum, so that it allows estimating oxidative activity.

When necessary, after incubation with the fluorochromes, an aliquot of embryo suspension was treated for $30 \mathrm{~min}$ with $100 \mu \mathrm{g}$ (f.c.) oligomycin or $1 \mathrm{mM}$ Sodium azide, which respectively inhibit ATP synthase (complex V of OXPHOS) and cytochrome c oxidase (complex IV).

\section{Microscopic observations}

For our observations we used a CLSM (Olympus FV-300) equipped with argon $(488 \mathrm{~nm})$ and helium/neon (543 nm) lasers. Several layers ( $2 \mu \mathrm{m}$ thick) of eggs and embryos were scanned with a PlanApo $60 \times 1.40$ oil-immersion lens at $1024 \times 1024$ pixel resolution. Specimens incubated with MTG absorb laser light at $490 \mathrm{~nm}$ wavelength emitting green fluorescent light at $516 \mathrm{~nm}$, those incubated with MTO absorb laser light at $551 \mathrm{~nm}$ emitting red fluorescent light at $576 \mathrm{~nm}$ wavelength.

Lasers specifically excite the single fluorochromes: blue laser at $488 \mathrm{~nm}$ excites MTG but not MTO. Vice versa, green laser at $543 \mathrm{~nm}$ excites MTO but not MTG, avoiding the false positive results.

\section{Image acquisition and analysis}

The CSLM fluorescence is revealed by photomultiplier tubes at 256 grey levels. As the acquisition software (OlympusFluoview v.3.3) links a different colour to each channel, green for MTG and red for MTO, we adopted the 8-bit values full range (0-255) with a linear Look Up Table in order to compare both colours.

Images obtained by CLSM were analyzed, measured and processed by the IMAGEJ software, a public domain Java Image processing program. The data obtained were elaborated by Microsoft Excel ${ }^{\mathrm{TM}}$.

\section{Results}

During oogenesis, sea urchin synthesizes and accumulates a large number of mitochondria, which are partitioned to the daughter cells during embryogenesis. It has been demonstrated that the number of maternal organelles is sufficient to support development until late stages without new synthesis of mitochondrial DNA or production of new mitochondria (Matsumoto et al., 1974; Rinaldi et al., 1979a,b).

In order to verify the mass, the distribution and the OXPHOS activity of mitochondria, we incubated eggs and embryos in vivo with two specific fluorochromes, MTG and MTO.

\section{Mitochondrial mass analysis}

In inverted CSLM, the laser beam excites the specimen from below and the emitted fluorescence is revealed by the photomultiplicator also located below. Consequently, the resulting fluorescence signals are gradually attenuated due 


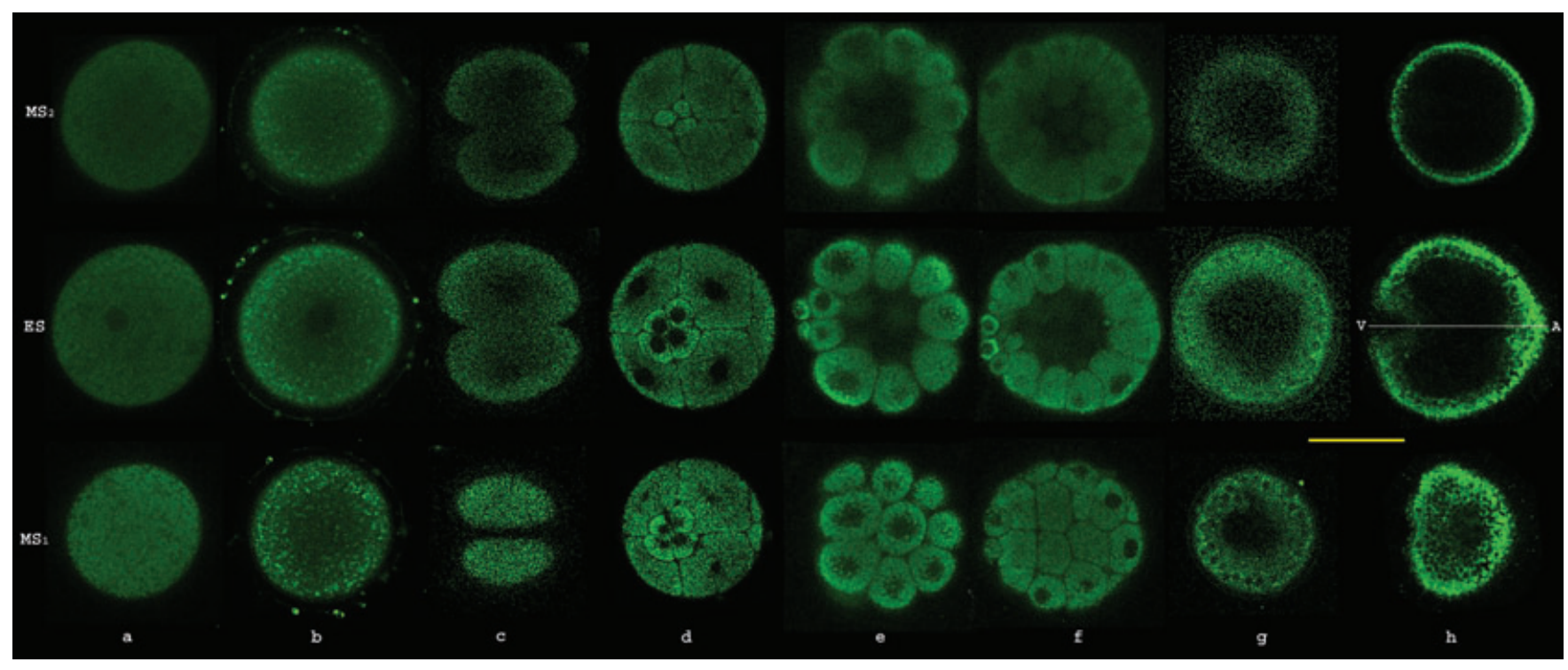

Fig. 1. Mitochondrial mass in P. lividus. Distribution of MTG in the equatorial section (ES), and middle sections (MS $\left.{ }_{1}, \mathrm{MS}_{2}\right)$ of unfertilized egg (a), justfertilized egg (b), two blastomeres (c), 16 blastomeres (d), 32 blastomeres (e), 64 blastomeres (f), blastula (g) and gastrula (h). Yellow bar $=50 \mu \mathrm{m}$, white line $=A-V$ axis.

to the distance of the light source from the sections of the specimen. Hence, images from the topmost layers are darker than the images captured from the lower layers, because of light-loss distortions (Capek et al., 2006). To show the distribution of organelles, we chose three main scanning sections: an equatorial section (Fig. 1, indicated as ES) and two parallel middle sections (Fig. 1, indicated as $\mathrm{MS}_{1}$ and $\mathrm{MS}_{2}$ ) in the upper and lower hemispheres. Figure 1 also shows the distribution of green in the sections of embryos at different developmental stages $(\mathrm{a}-\mathrm{h})$.

However, microscopic observations were performed capturing $2 \mu \mathrm{m}$ thick layers of eggs and embryos, the number of the captured sections turns out to be a total of 30 in all cases. Of the several thousands embryos we observed, 20 for all developmental stage were analyzed and processed. In order to calculate the total mitochondrial mass we integrated the values of pixel intensities for all captured sections. The arithmetic mean of these sums was used to draw the histogram in Fig. 2, which shows relatively constant values of intensities, indicating a fairly constant mitochondrial mass, suggesting that the amount of organelles does not change during development.

\section{Mitochondrial activity analysis}

Mitochondrial oxidative activity was monitored as shown in Fig. 3. Incubation with MTG and MTO fluorochromes can simultaneously allow measuring mitochondrial mass and activity. The intensity of green is proportional to the mitochondrial mass, whereas the intensity of red is proportional to mitochondrial activity, so that red pixels are a subset of green pixels. If the intensities of green and red pixels are similar the resulting merged colour is yellow, whereas in the regions of higher oxygen consumption the colour verges towards red. Figure 4 shows that mitochondria of unfertilized eggs (a) consume oxygen, but the consumption is not very high (uniform yellow), and that mitochondrial activity increases after fertilization (b, c), maintaining quite the same distribution. At 16, 32, 64 blastomeres, micromeres show higher oxygen consumption than the other blastomeres (d, e, f). At blastula (g), mitochondrial activity is higher around the blastocoel, especially in the region corresponding to the vegetal plate. At gastrula $(\mathrm{h})$, the region corresponding to the blastopore and the cells in the archenteron cavity (mesenchyme cells) contain more respiring organelles.

In order to ascertain whether the red fluorescence was due to oxygen produced by OXPHOS, we treated eggs and embryos with specific inhibitors of mitochondrial complexes, oligomycin (inhibitor of $\mathrm{F}_{1}-\mathrm{F}_{0}$ ATP synthase) and sodium azide (inhibitor of cytochrome $c$ oxidase). The treatments strongly reduced the red but not the green fluorescence (Fig. 5).

\section{Image analysis}

Of the several thousands of embryos we observed, we chose 20 embryos oriented as shown in Fig. 4. The images captured with the confocal microscope were then analyzed and the fluorescence intensities were measured using IMAGEJ. We obtained tables of the positions along the $A-V$ axis, indicated as $P$ in the graphs, and the corresponding intensities $I$, i.e. the measures of the mean value intensity of all the points lying on the normal to the $A-V$ axis, for both green and 


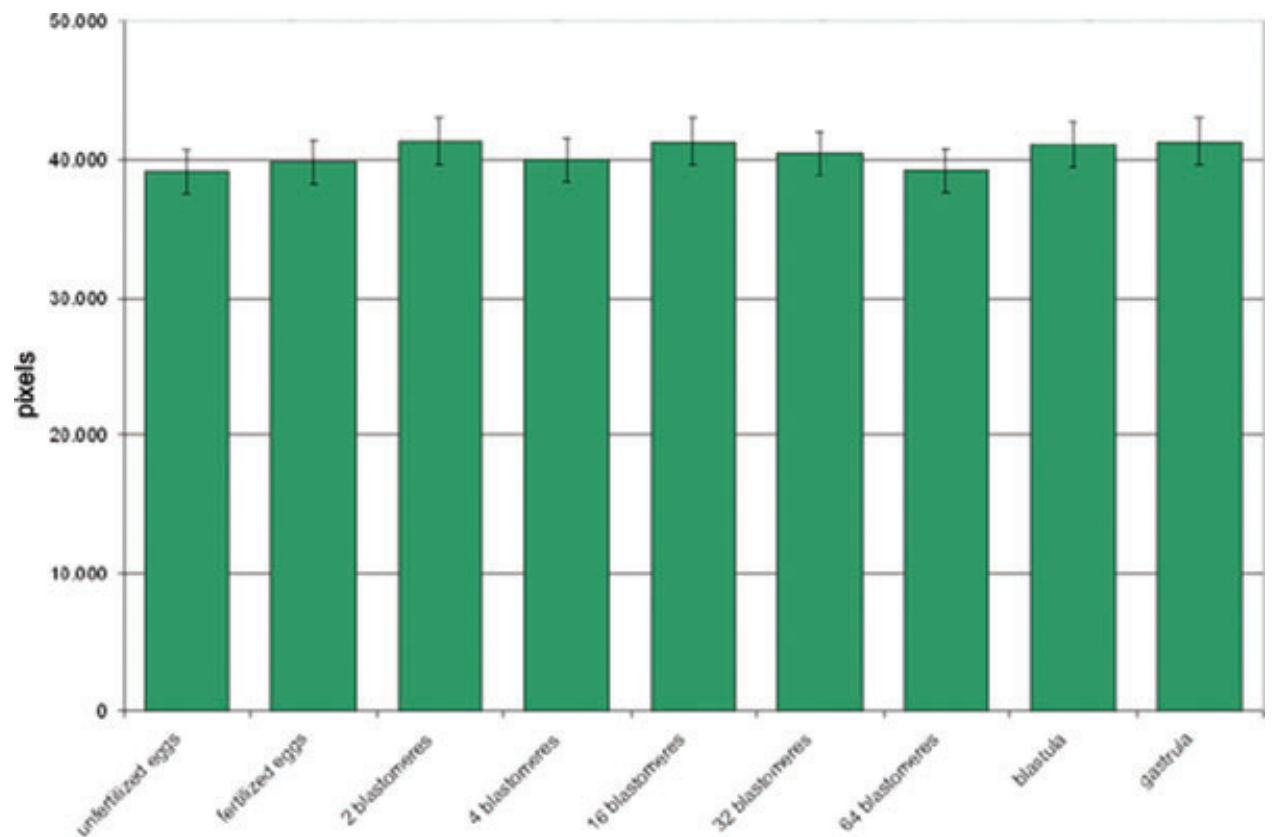

Fig. 2. Mitochondrial mass during development. Abscissa: developmental stages; ordinate: integration of pixel intensity values of all captured sections. Bars indicate the standard deviations.

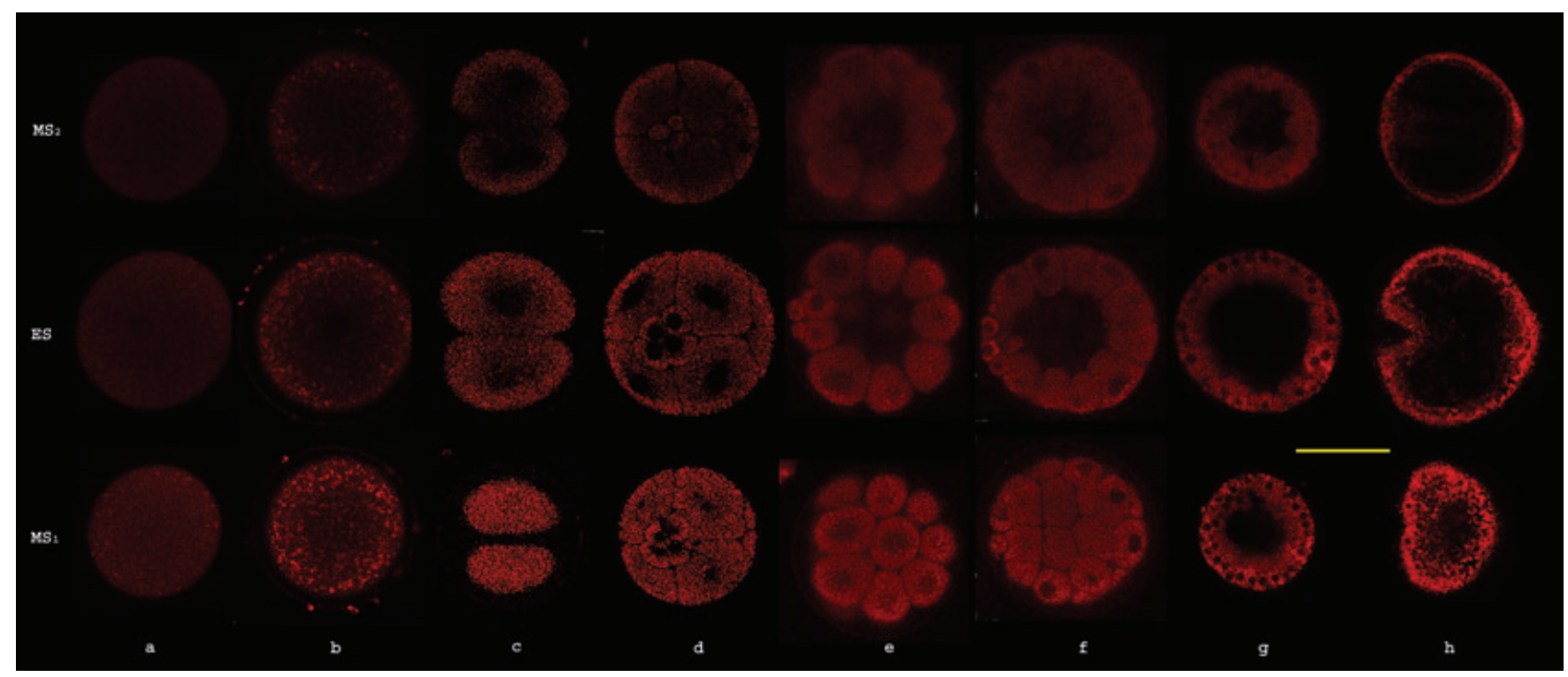

Fig. 3. Mitochondrial oxidative activity. Distribution of MTO in the sections ES, $\mathrm{MS}_{1}, \mathrm{MS}_{2}$ of unfertilized egg (a), just-fertilized egg (b), two blastomeres (c), 16 blastomeres (d), 32 blastomeres (e), 64 blastomeres (f), blastula (g) and gastrula (h). Bar $=50 \mu \mathrm{m}$.

red values. These tables were used to draw graphs of $I_{\mathrm{g}}$ and $I_{\mathrm{r}}$ (the intensity of green and red, scaled from 0 to 255) as function of $P$ in pixels (Fig. 6). In these graphs, the areas under the curves represent the measures of mitochondrial mass and mitochondrial activity, respectively. The area under red intensity curves, corresponding to the mitochondrial activity referred to in each stage, increases during development. It is noteworthy that, although in the early embryos active mitochondria are uniformly distributed (Figs 4 and $6 \mathrm{~b}-\mathrm{d}$ ), at later stages the micromeres (e, f) display a higher oxygen consumption than macromeres and mesomeres. In addition, the cells of the blastula vegetal plate $(\mathrm{g})$ and of blastopore region of gastrula (h) are respiring at higher level than in the rest of the embryos, suggesting an increasing oxidative phosphorylation capacity of mitochondria in these embryo regions. 


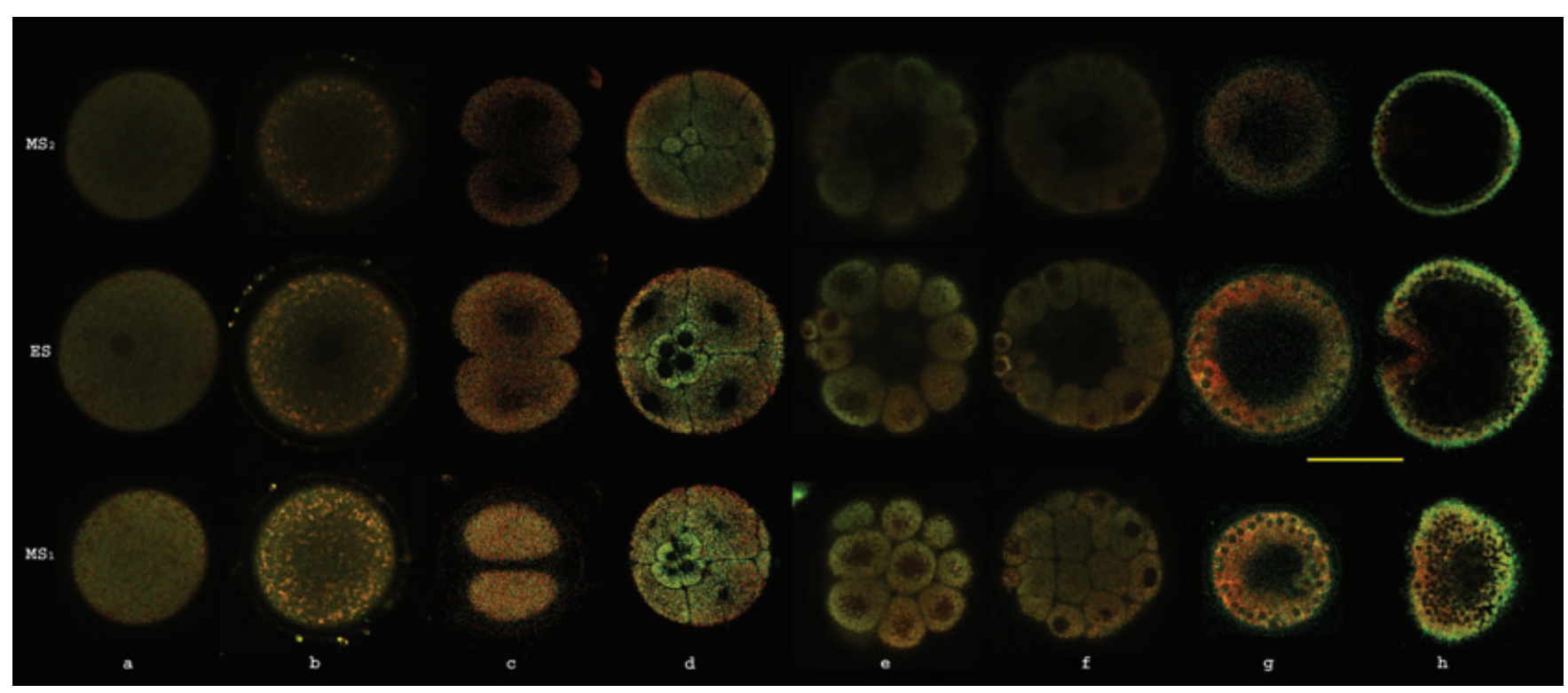

Fig. 4. Mitochondrial mass and oxidative activity. Merge of green (MTG) and red (MTO) fluorescence in the sections ES, MS $1, \mathrm{MS}_{2}$ of unfertilized egg (a), fertilized egg (b), two blastomeres (c), 16 blastomeres (d), 32 blastomeres (e), 64 blastomeres (f), blastula (g) and gastrula (h). Bar $=50 \mu \mathrm{m}$.

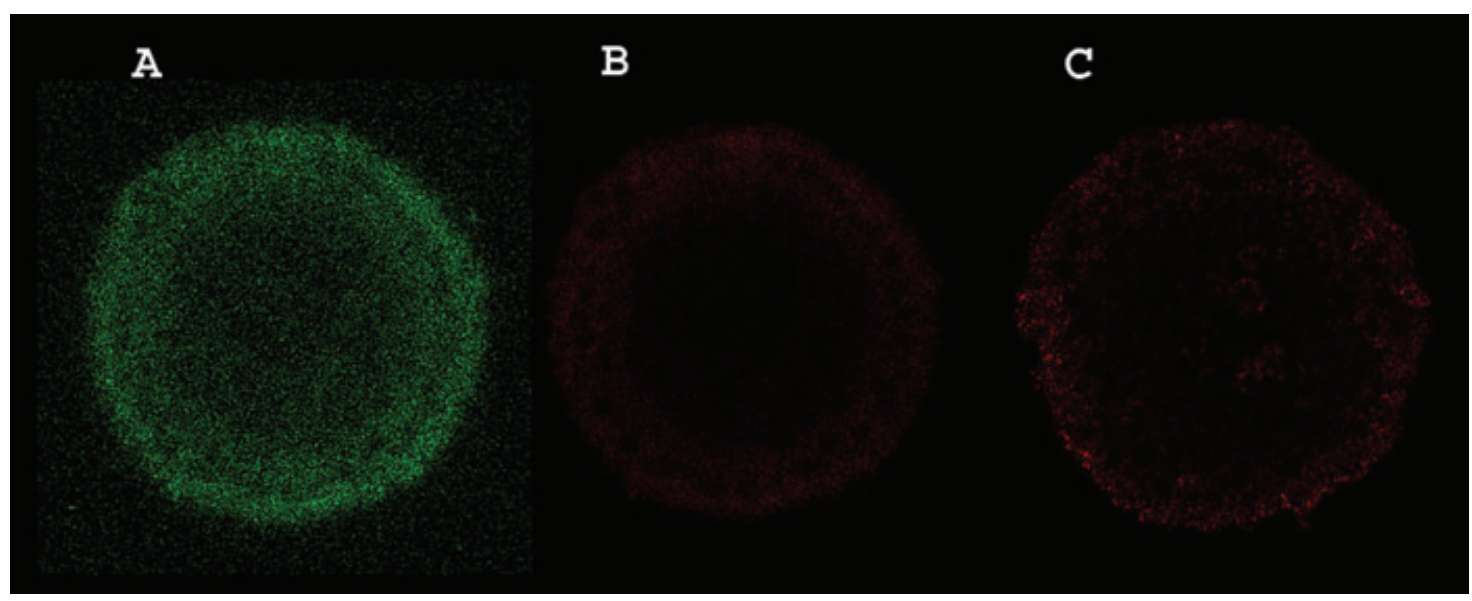

Fig. 5. Effects on P. lividus blastula of treatment with oligomycin or sodium azide. Mitochondrial mass (A) and oxidative activity after oligomycin (B) and sodium azide $(C)$ treatments.

\section{Analysis of intensity variations}

Each experiment was carried out varying laser voltage, in order to obtain a rather intense signal and avoiding peak values over 255 (saturation), for both green and red channels. To avoid small scattering ripples in the intensity data, we adopted a median filter that substituted each intensity value with the average of its surrounding (for values ranging from -10 to +10 of the median). Finally, from these filtered values, we elaborated a variation diagram of relative intensity $|\Delta I / \Delta P|$ in function of $P$ along the $A-V$ axis. We chose the absolute values so that the resulting curves could be easily compared.

Figure 7 shows the variation curves, along the $A-V$ axis, of the green fluorescence during development. The green curves closely resemble straight lines with the same slope, suggesting that the amount of mitochondria is substantially constant during development. Figure 8 shows the variation curves of the red fluorescence. It is noteworthy that the slope of the red variation curves increases from unfertilized egg to the gastrula stage, suggesting that mitochondrial activity increases during development. It is interesting to observe that, at blastula and gastrula, the slope is not constant, with a different shape in some regions.

\section{Variation index}

Next, by using a linear equation, we fitted the variation curves to the data, then we calculated the slope values for each line, 

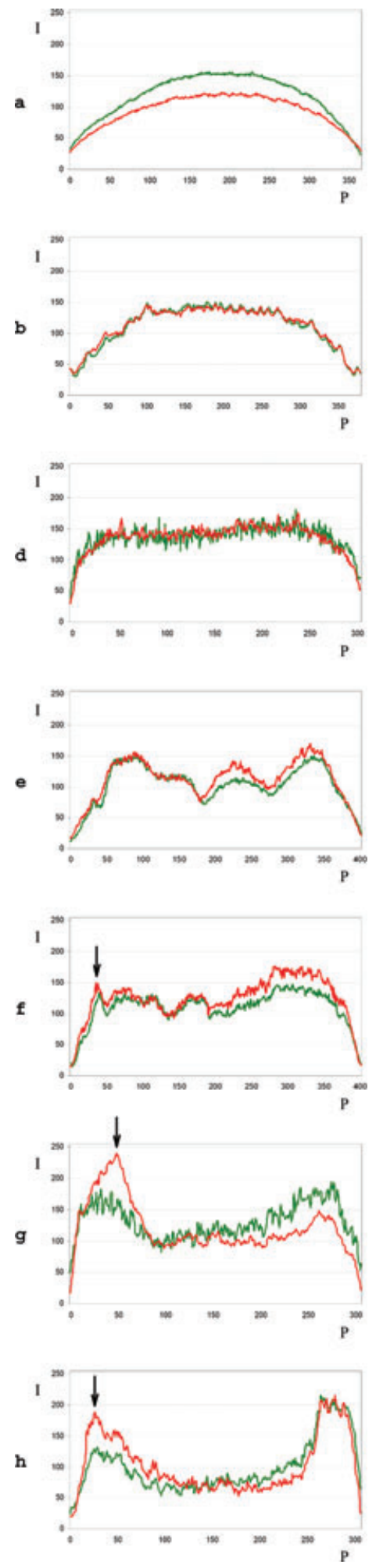

Fig. 6. Intensity of MTG and MTO in the equatorial section (ES) of unfertilized egg (a), fertilized egg (b), 16 blastomeres (d), 32 blastomeres (e), 64 blastomeres (f), blastula (g), and gastrula (h). Abscissa: position values $(P)$ along $A-V$ axis; ordinate: normalized intensity values $(I)$; the curves $I_{\mathrm{g}}$ and $I_{\mathrm{r}}$ correspond, respectively, to MTG and MTO intensities. Arrows indicate the position of the cells at vegetative pole.

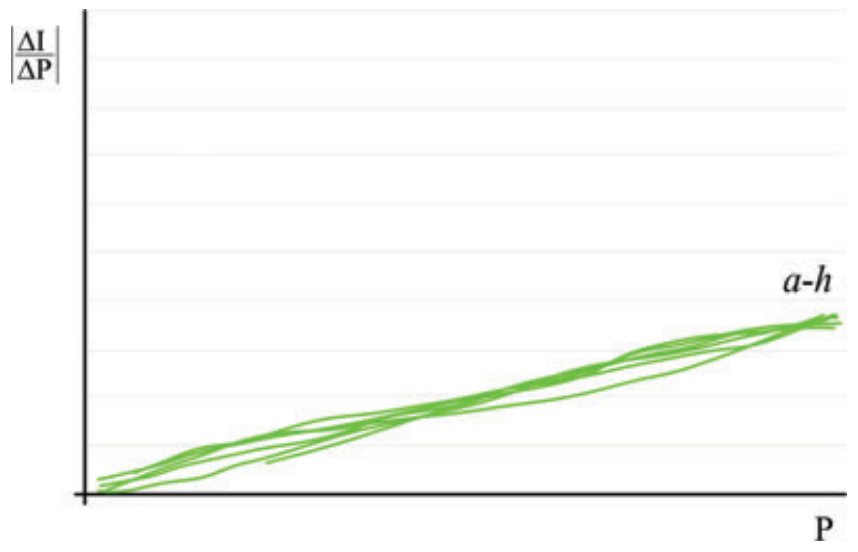

Fig. 7. Intensity variation of mitochondrial mass during development: egg (a), fertilized egg (b), two blastomeres (c), 32 blastomeres (e), 64 blastomeres (f), blastula $(\mathrm{g})$, gastrula $(\mathrm{h})$. Abscissa: position values $(P)$ along $A-V$ axis; ordinate: relative intensity variation $|\Delta I / \Delta P|$.

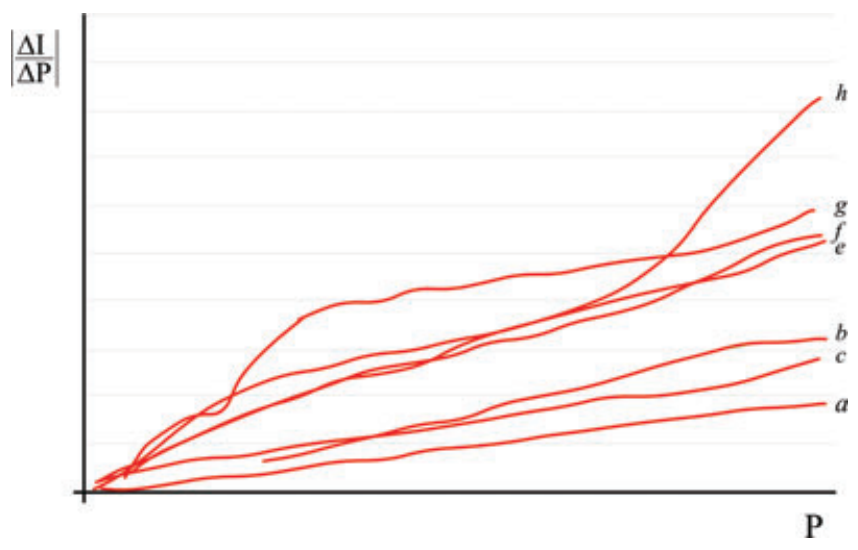

Fig. 8. Intensity variation of mitochondrial oxidative activity during development: egg (a), fertilized egg (b), two blastomeres (c), 32 blastomeres (e), 64 blastomeres (f), blastula (g), gastrula (h). Abscissa: position values $(P)$ along $A-V$ axis; ordinate: relative intensity variations $|\Delta I / \Delta P|$.

obtaining the arctangent values, as shown in Fig. 9. The arctangent values at different stages, define the variation index relative to mitochondrial mass and mitochondrial activity during sea urchin development. The data obtained from this analysis show that the green index has a constant value during development, confirming the results, obtained by integration method reported in Fig. 2, that the mitochondrial mass does not vary during development. It is very interesting that the red index, which indicates oxygen consumption, changes during development, showing a sharp increase after fertilization, at 32 blastomeres and at blastula stage.

\section{Discussion}

It is well known that in sea urchin, during oogenesis, mitochondria are synthesized and accumulated in a very high number, and that after fertilization they are partitioned 


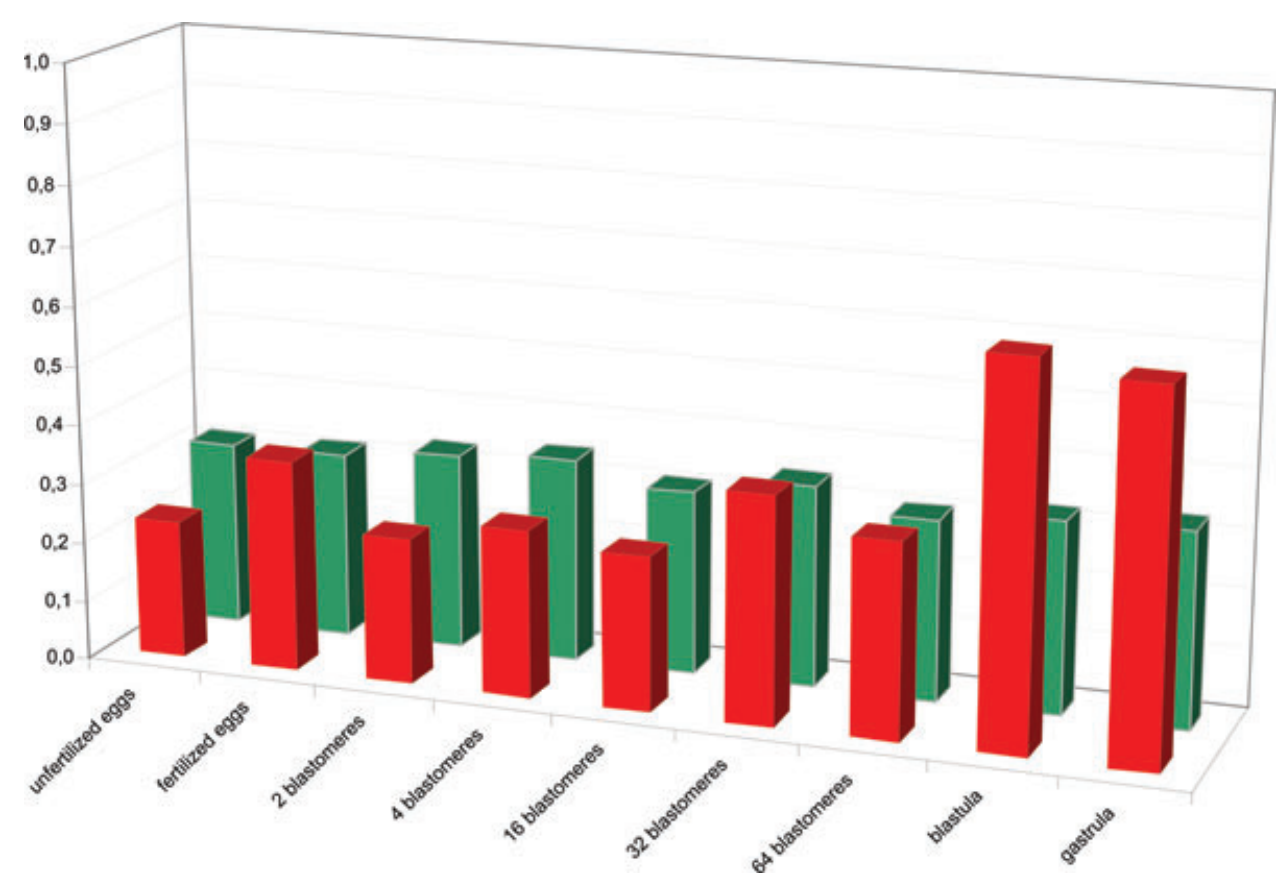

Fig. 9. Variation index of mitochondrial mass (green) and activity (red) during development. Abscissa: stages of development; ordinate: arctangent value relative to the slopes of variation curves for MTG and MTO.

to daughter cells. Then, the organelles of maternal origin are sufficient to support the energetic need of the embryos until very late stages of development, without synthesis of new mitochondria or mitochondrial DNA (Matsumoto et al., 1974; Rinaldi et al., 1979a,b). After fertilization, the respiratory rate of sea urchin embryos increases with a burst of oxygen consumption $1 \mathrm{~min}$ after fertilization, reaching a value 15 times higher than that of unfertilized egg (see Giudice, 1985, for extensive review). After this initial burst, oxygen consumption decreases and reaches a level higher than that of unfertilized eggs. Then respiration keeps quite constant up to blastula, slightly increases after hatching, reaches the maximum value at gastrula stage and then it is maintained at a high level up to pluteus (see Fujiwara \& Yasumasu, 1997, for extensive review). The reason why this happens has been investigated and extensively debated. Fujiwara \& Yasumasu (1997) suggested that electron transport of mitochondrial respiratory chain is regulated by ADP control and by limitations of cytochrome $c$ reduction in early embryos, and that late gastrulae are released from this control.

It was also well demonstrated that, in sea urchin, respiration depends solely on the mitochondrial electron transport machinery (Yasumasu et al., 1984, 1996; Fujiwara \& Yasumasu, 1997). The electron transport and the amount of mitochondrial proteins are similar before and after fertilization (Selak \& Scandella, 1997; Yasumasu et al., 1996) and during early development (Fujiwara \& Yasumasu, 1997; Fujiwara et al., 2000).
In this work, we investigated the number, the distribution and the respiratory activity of mitochondria, by incubating $P$. lividus eggs and embryos with the fluorescent probes MTG FM, to measure the mass and distribution, and MTO CM- $\mathrm{H}_{2}$ TMRos, to measure oxygen consumption.

Treatment of eggs and embryos with specific inhibitors of mitochondrial complexes, oligomycin (inhibitor of $\mathrm{F}_{1}-\mathrm{F}_{0}$ ATP synthase), and sodium azide (inhibitor of cytochrome $c$ oxidase), strongly reduced respiratory activity, as demonstrated by the disappearance of the red but not of the green fluorescence.

In order to validate the results derived from microscopic observations, we elaborated a mathematic discrete model in relation to the experimental data. By this mean, we measured the intensities of both fluorochromes in the different sections of the same embryo, and calculated the intensity variations and the variation index.

The obtained data suggest that mitochondrial mass is constant during development, and respiratory activity is enhanced at fertilization and increases until gastrula. It is noteworthy that mitochondrial activity, uniform in the embryo cells during early development, becomes higher in the micromeres. Indeed, it has been demonstrated that, in sea urchin embryos, micromeres have distinct functions: they differentiate autonomously into the skeletogenic mesenchyme cells, induce endomesoderm formation (Yazaki et al., 2004; Kurihara \& Amemiya, 2005; Yamazaki et al., 2005), and act as a morphogenetic centre, releasing regulatory signals for the specification of cell fates along the animal-vegetal and 
oral-aboral axes (Oliveri et al., 2002, 2003; Kitazawa \& Amemiya, 2007).

In addition, our results demonstrate that the cells of the blastula vegetal plate and of blastopore region of gastrula are respiring at higher level than the cells of the rest of the embryo, suggesting an increasing oxidative phosphorylation capacity of mitochondria. This can be justified admitting that the cells in the vegetal plate, or those of the blastopore, are probably doing extra work, because they are duplicating and migrating at the same time, dragging the surrounding cells.

This observation is not surprising, because in some animal phyla, in different systems and in many different cell types, morphological and functional differences between mitochondria were previously underlined, even in the same individual. For example, in Xenopus embryos, the activity of enzymes in the mitochondrial matrix varies with the developmental stage and with the tissue type (Kistler \& Weber, 1974). In early Drosophila embryos, respiratory activity of mitochondria is higher in the polar plasm than in the other regions of periplasm, and changes during development (Akiyama \& Okada, 1992). In the embryos of mouse and slug (Arion ater rufus), a remarkable spatiotemporal heterogeneity in the fine structure of mitochondria was reported (Sathananthan, 1970; Stern et al., 1971).

\section{Acknowledgements}

We thank S. Oddo and S. Arione for critical reading of the manuscript. This work was supported by MIUR (ex 60\% and COFIN 2004, grant 2004057282-003).

\section{References}

Akiyama, T. \& Okada, M. (1992) Spatial and developmental changes in the respiratory activity of mitochondria in early Drosophila embryos. Development 115, 1175-1182.

Bresch, H. (1978) Mitochondrial profile densities and areas in different developmental stages of the sea urchin Sphaerechinus granularis. Exp. Cell. Res. 111, 205-209.

Buttino, I., Ianora, A., Carotenuto, Y., Zupo, V. \& Miralto, A. (2003) Use of the confocal laser scanning microscope in studies on the developmental biology of marine crustaceans. Micros. Res. Tech. 60, 458-464.

Cameron, R.A., Fraser, S.E., Britten, R.J. \& Davidson, E.H. (1989) The oralaboral axis of sea urchin embryo is specified by first cleavage. Development 106, 641-647.

Capek, M., Janacek, J. \& Kubinova L. (2006) Methods for compensation of the light attenuation with depth of images captured by a confocal microscope. Microsc. Res. Tech. 69, 624-635.

Coffman, J.A. \& Davidson, E.H. (2001) Oral-aboral axis specification in the sea urchin embryo. I. Axis entrainment by respiratory asymmetry. Dev. Biol. 230, 18-28.

Coffman, J.A., McCarthy, J.J., Dickey-Sims, C. \& Robertson, A.J. (2004) Oral-aboral axis specification in the sea urchin embryo. II. Mitochondrial distribution and redox state contribute to establishing polarity in Strongylocentrotus purpuratus. Dev. Biol. 273, 160-171.
Davidson, E.H., Cameron, R.A. \& Ransick A. (1998) Specification of cell fate in sea urchin embryo: summary and some proposed mechanisms. Development 125, 3269-3290.

Fujiwara, A. \& Yasumasu, I. (1997) Does the respiratory rate in sea urchin embryos increase during early development without proliferation of mitochondria? Dev. Growth Differ. 39, 179-189.

Fujiwara, A., Kamata, Y., Asami, K. \& Yasumasu, I. (2000) Relationship between ATP level and respiratory rate in sea urchin embryos. Dev. Growth Differ. 42, 155-165.

Giudice G. (1985) The Sea Urchin Embryo, pp. 73-74. Springer-Verlag, Berlin.

Hertzler, P.L. \& Clark, W.H. Jr (1992) Cleavage and gastrulation in the shrimp Sicyonia ingentis: invagination is accompanied by oriented cell division. Development 116, 127-140.

Holy, J.M. (1999) Imaging sea urchin fertilization. Methods in Molecular Biology, Confocal Microscopy Methods and Protocols (ed. by S. W. Paddock), pp. 153-166. Humana Press, Totowa, NJ.

Kistler, A. \& Weber, R. (1974). Enzyme patterns in mitochondria of eggs, liver, and skeletal muscle during larval development of Xenopus. Dev. Biol. 37, 236-247.

Kitazawa, C. \& Amemiya, S. (2007) Micromere-derived signal regulates larval left-right polarity during sea urchin development. J. Exp. Zool. Part A Ecol. Genet. Physiol. 307A, 249-262.

Kurihara, H. \& Amemiya, S. (2005) Developmental potential of small micromeres in sea urchin embryos. Zoolog. Sci. 22, 845852.

Matsumoto, L., Kasamatsu, H., Pikó, L. \& Vinograd, J. (1974) Mitochondrial DNA replication in sea urchin oocytes. J. Cell Biol. 63 , 146-159.

Oliveri, P., Carrick, D.M. \& Davidson, E.H. (2002) A regulatory gene network that directs micromere specification in the sea urchin embryo. Dev. Biol. 246, 209-228.

Oliveri, P., Davidson, E.H. \& McClay, D.R. (2003) Activation of pmar1 controls specification of micromeres in the sea urchin embryo. Dev. Biol. 258, 32-43.

Pawley, J.B. (eds) (1995) Handbook of Biological Confocal Microscopy, 2nd edn. Plenum Press, New York.

Rinaldi, A.M., De Leo, G., Arzone, A., Salcher, I., Storace, A. \& Mutolo, V. (1979a) Biochemical and electron microscopic evidence that cell nucleus negatively controls mitochondrial genomic activity in early sea urchin development. Proc. Natl. Acad. Sci. U.S.A. 76, 1916-1920.

Rinaldi, A.M., Salcher-Cillari, I. \& Mutolo, V. (1979b) Mitochondrial division in non nucleated sea urchin eggs. Cell. Biol. Int. Rep. 3, 179182.

Sathananthan, A.H. (1970). Studies on mitochondria in the early development of the slug Arion ater rufus L. J. Embryol. Exp. Morphol. 24, 555-582.

Selak, M.A. \& Scandella, C.J. (1987). Respiration capacity of mitochondria isolated from unfertilized and fertilized sea urchin eggs. Exp. Cell Res. $169,369-378$.

Stern, S., Biggers, J.D. \& Anderson, E. (1971) Mitochondria and early development of the mouse. J. Exp. Zool. 176, 179-191.

Summers, R.G., Stricker, S.A. \& Cameron, R.A. (1993) Applications of confocal microscopy to studies of sea urchin embryogenesis. Methods in Cell Biology: Cell Biological Applications of Confocal Microscopy (ed. by B. Matsumoto), pp. 266-286. Academic Press, San Diego.

Yamazaki, A., Kawabata, R., Shiomi, K., Amemiya, S., Sawaguchi, M., Mitsunaga-Nakatsubo, K. \& Yamaguchi, M. (2005) The micro1 gene is necessary and sufficient for micromere differentiation and 
mid/hindgut-inducing activity in the sea urchin embryo. Dev. Genes Evol. 215, 450-459.

Yasumasu, I., Fujiwara, A., Hino, A. \& Asami, K. (1984) Effect of several redox dyes on the respiration of unfertilized egg of sea urchin. Zool. Sci. $1,725-736$.

Yasumasu, I., Tazawa, E., Asami, K. \& Fujiwara, A. (1996) Does the low respiratory rate in unfertilized eggs result mainly from depression of the redox reaction catalyzed by flavoproteins? Analysis of the respiratory system by light-induced release of CO-mediated inhibition. Dev. Growth Differ. 38, 359-371.

Yazaki, I., Abe, M., Santella, L. \& Koyama, Y. (2004) Mechanisms of calcium elevation in the micromeres of sea urchin embryos. Biol. Cell 96, 153-167.

Zhang, W.H., Zhu, S.N., Lu, S.L., Huang, Y.L. \& Zhao, P. (2000). Threedimensional image of hepatocellular carcinoma under confocal laser scanning microscope. World J. Gastroenterol. 6, 344-347. 